\title{
Otoplastia en orejas prominentes: alternativa quirúrgica
}

\section{Otoplasty in prominent ears: surgical alternative}

\author{
Dávalos Dávalos, P.*, Ramírez Rivera, J .I.**, Dávalos Dávalos, P.A.***
}

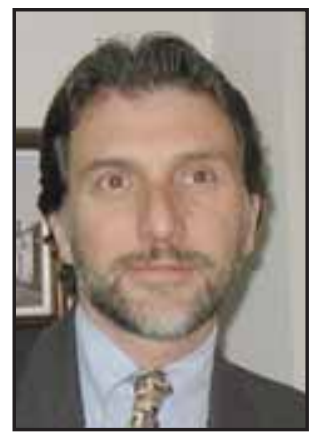

Dávalos Dávalos, $\mathrm{P}$.

\section{Resumen}

Las orejas prominentes o en asa son la deformidad congénita más frecuente del pabellón auricular. En esta patología es notoria la falta del antihélix y el aumento del ángulo mastoideoconchal, teniendo en cuenta que las dimensiones del área del pabellón auricular estén dentro de unos límites normales.

Han sido descritas numerosas y diversas técnicas quirúrgicas para su corrección, buscando todas ellas cómo acortar el ángulo mastoideo conchal y crear un nuevo antihélix. Cada oreja tiene sus propias características en el contexto anatómico normal del paciente, por lo que es importante realizar una evaluación correcta para aplicar la técnica quirúrgica más adecuada a cada caso.

En el presente trabajo describimos sistemáticamente los pasos quirúrgicos a seguir buscando la simplificación del procedimiento y sobre todo, demostrando que con una incisión mínima producida por una aguja calibre $21 \mathrm{G}$ con punta angulada, mediante la que se realizan cortes de relajación en la superficie anterior del cartílago paralelos al eje vertical del pabellón auricular en el área que corresponde a la proyección del antihélix, se puede obtener una curvatura natural del neoantihélix.
Prominent ear, is the most frequent congenital malformation of the auricular pavillion. In this pathology, the absence of the antihelix is notorious as well as the increase of the mastoid-conchal angle, considering that the dimensions of the area of the auricular pavillion are within the normal limits. Many techniques have been described, all of them trying to reduce the mastoid-conchal angle and to create a new antihelix. Each ear has its own characteristics within the normal anatomic context; so it is important to make a correct evaluation in order to apply the adequate surgical technique. The surgical steps to be followed are systematically described to simplify the procedure, and most of all, showing that with a minimum incision done with a $21 \mathrm{G}$ size angulated needle, over the anterior side of the auricular cartilage, we can perform relaxation cuts parallel to the vertical axe of the auricular pavillion, and obtain a natural curve of the neoantihelix.

\author{
Palabras clave Orejas prominentes, Orejas protuidas, \\ Orejas en asa, Orejas en soplillo, Oto- \\ plastia, Neoantihélix \\ Código numérico 254-2546
}

\begin{tabular}{|ll|}
\hline Key words & $\begin{array}{l}\text { Prominent ear, Protuded ear, Ear in han- } \\
\text { dle, Puff ear, Otoplasty, Neoantihelix }\end{array}$ \\
Numeral Code & $254-2546$
\end{tabular}

\footnotetext{
* J Jefe de Cirugía Plástica y Unidad de Quemados Hospital Pediátrico Baca Ortíz, Quito, Ecuador. Profesor de Postgrado de Cirugía Plástica, Universidad San Francisco de Quito. Profesor Postgrado Cirugía Universidad Internacional de Ecuador.

* Médico Postgrado de Cirugía Pediátrica Hospital Pediátrico Baca Ortiz, Universidad Internacional de Ecuador. Quito-Ecuador.

* Médico Residente, Instituto de Cirugía Plástica Dávalos. Quito, Ecuador.
} 


\section{Introducción}

El pabellón auricular forma parte del oído externo y tiene como propósito captar y direccionar las ondas sonoras hacia el conducto auditivo. Está formado por un esqueleto de cartílago elástico con formas definidas, cubierto en su totalidad por piel y situado en la pared lateral de la cabeza, unido a ella por su parte media y libre en el resto de su extensión (1). La ubicación anatómica del pabellón auricular está entre una línea horizontal superior que coincide con la parte más lateral de la ceja y otra inferior, un poco más abajo del septum caudal. Su eje mayor, mide generalmente 60 a $65 \mathrm{~mm}$ y su eje transversal 25 a $35 \mathrm{~mm}(2,3)$.

Las orejas prominentes, protuídas (4), en soplillo o en asa (Fig. 1), son una patología congénita que produce un efecto psicológico de preocupación o complejo en el paciente y/o en su entorno familiar. Son la deformidad auricular más frecuente $(5,6)$ del pabellón auricular; aparecen en aproximadamente el 5\% de la población $(7,8)$ y constituyen uno de los problemas congénitos más comunes en Cirugía Plástica (9). Esta patología se asocia frecuentemente a problemas psicológicos y trastornos emocionales en niños y adultos $(6,10)$, llegando a crear complejo de inferioridad en muchos casos (11).

Su forma más frecuente en la de valgus de la concha, con formación de un ángulo entre el pabellón auricular y la mastoides mayor de $45^{\circ}$ (12), llegando en la mayoría de los casos a $90^{\circ}$, con pobre plegamiento o ausencia del antihélix y raramente con hipertrofia de la concha $(5,6,8)$.

En esta deformidad, el tamaño de la oreja corresponde al desarrollo corporal del paciente, con crecimiento rápido en los primeros dos años de edad hasta alcanzar aproximadamente $5 \mathrm{~cm}$ de su eje vertical; a partir de ese momento su crecimiento se torna lento, alcanzando su maduración en torno a los 17 años, cuando la oreja presenta una longitud de su eje vertical de 6 a $6,5 \mathrm{~cm}(5)$.

La afectación suele ser bilateral y puede diferir entre ambas orejas (6). Ocasionalmente se pueden observar orejas alargadas que protruyen sin presentar las anomalías antes mencionadas (11).

El tratamiento de esta malformación es un procedimiento quirúrgico diseñado para dar a la oreja una apariencia más natural y anatómica (13).

Aunque es fundamental una correcta valoración de cada deformidad en cada paciente, cualquier intento de corrección quirúrgica basado solo en ángulos y medidas daría como consecuencia una forma poco natural de la oreja (5). Siendo numerosas las técnicas quirúrgicas descritas y empleadas para la corrección de las orejas prominentes, el cirujano debe utilizar

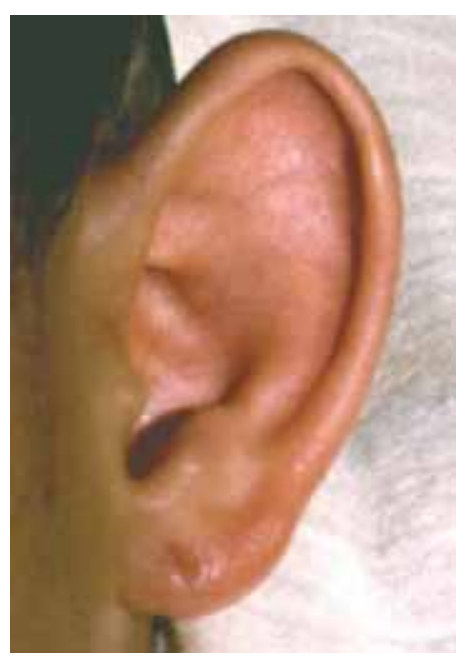

Fig. 1. Oreja prominente típica.

aquella con la que mejores resultados obtenga.

En el presente artículo describimos una técnica de otoplastia para el tratamiento de orejas prominentes basada en técnicas descritas anteriormente a las que aportamos una simplificación del procedimiento quirúrgico mediante la utilización de un instrumento simple, una aguja calibre $21 \mathrm{G}$ modificada con la que, a través de una incisión mínima, se realizan varios cortes de relajación en el cartílago paralelos al eje vertical del pabellón auricular en el área de proyección que correspondería al antihélix, evitando lesionar los tejidos adyacentes y consiguiendo fácilmente la formación del neoantihélix y de un ángulo mastoideo conchal de $30^{\circ}$, que le otorgan al pabellón auricular una apariencia de normalidad y de simetría entre ambas orejas.

Este procedimiento quirúrgico es fácil de ejecutar, sin mayor complejidad técnica y demanda poco tiempo operatorio $(12,14)$.

\section{Material y método}

\section{Técnica quirúrgica}

Realizamos el procedimiento bajo anestesia general o con bloqueo local con Xilocaína al 2\% con Epinefrina al 1:200000.

Comenzamos marcando en la piel la futura zona anatómica que corresponderá al neoantihélix, creado mediante una ligera presión digital a nivel del tubérculo de Darwin hacia el cráneo (Fig. 2,3).

Realizamos una transfixión empleando Azul de Metileno con aguja $\mathrm{N}^{\circ}$ 24G a nivel del borde de la marcación del neoantihélix, para imprimir sobre el plano auricular posterior la marcación del neoantihélix (Fig. 4, 5).

Marcamos los puntos en el borde externo posterior, respetando la piel propia del hélix (Fig. 6, 7). Seguidamente marcamos también el borde interno posterior a 5 mm del vértice del ángulo mastoideo-conchal (Fig. 8). 

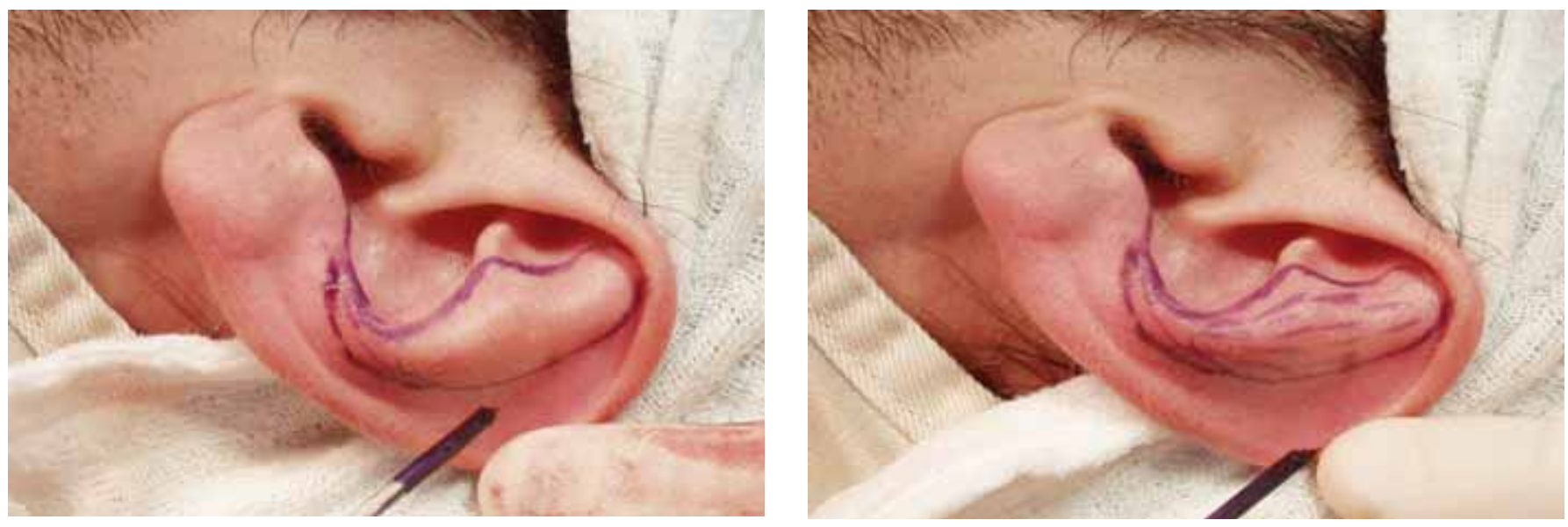

Fig. 2-3. Marcación del neoantihélix.
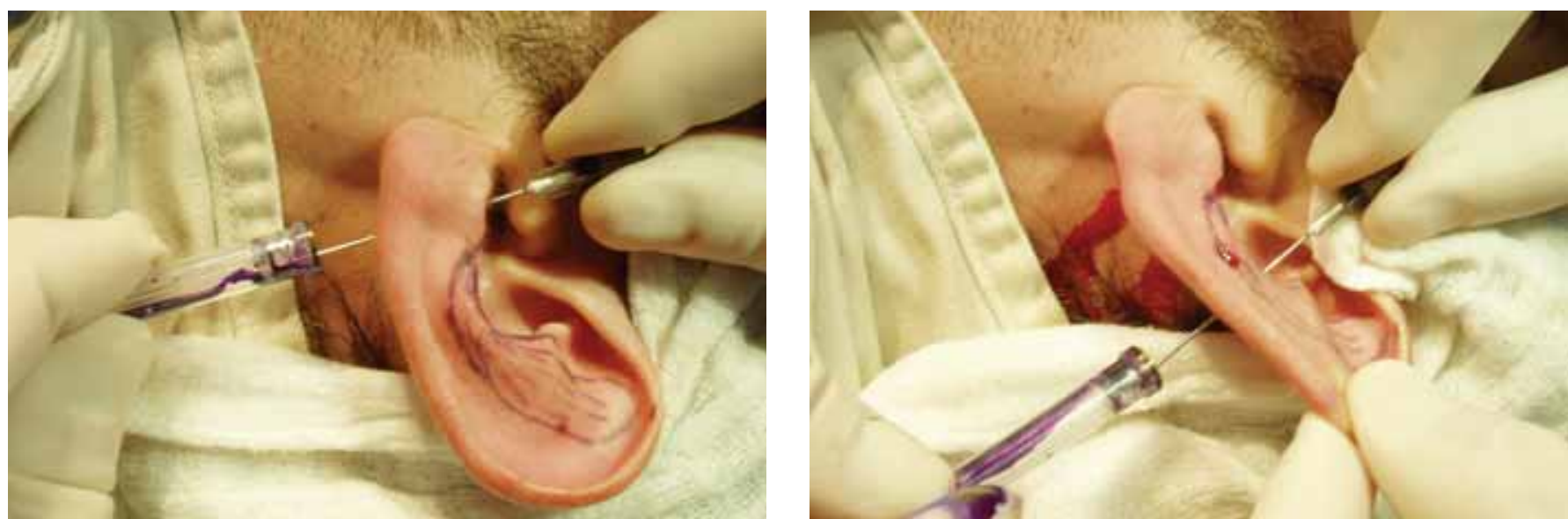

Fig. 4-5. Transfixión con Azul de metileno para imprimir en el plano posterior auricular el marcado del neoantihélix.

Realizamos un hidrodecolamiento con Xilocaína al 2\% con Epinefrina al 1:200000 dentro del área marcada en el pabellón auricular posterior (Fig. 9) y procedemos con bisturí $\mathrm{N}^{\circ} 15$ a incidir en la marcación interna. A continuación trazamos la incisión en la marcación externa del pabellón auricular (Fig.10) y resecamos piel y tejido celular subcutáneo en forma de huso en las áreas delimitadas hasta llegar al pericondrio (Fig. 11). Realizamos hemostasia cuidadosa

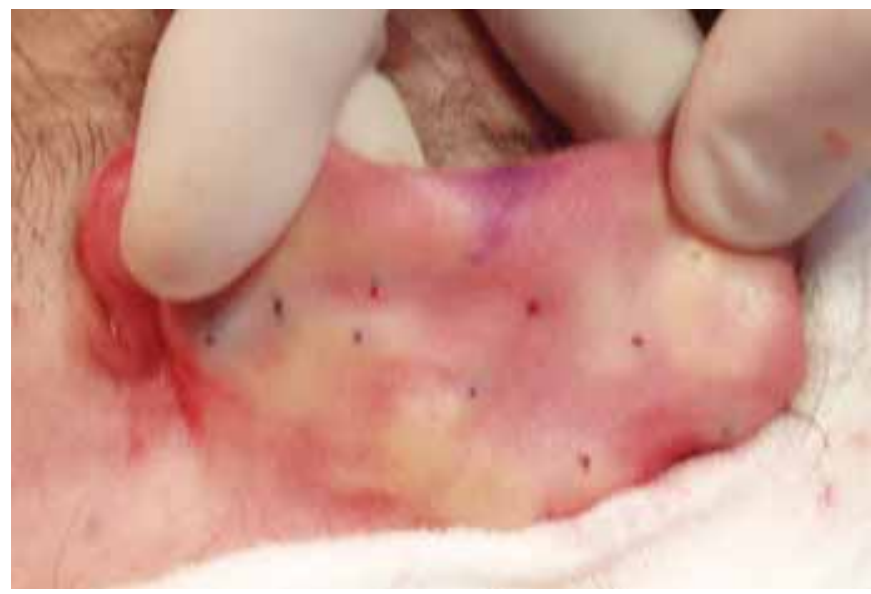

Fig. 6-7. Marcaje del borde externo posterior sobre la piel del hélix. en el pericondrio-cartílago auricular posterior expuesto (Fig. 12).

Seguidamente procedemos nuevamente a una infiltración subcutánea con Xilocaína al 2\% con Epinefrina al 1:200000 para producir hidrodecolamiento también en el espacio cutáneo-pericondrial correspondiente al área del antihélix en el pabellón auricular anterior, lo que nos facilita la separación de la piel anterior del pabellón auricular del cartílago (Fig. 13).

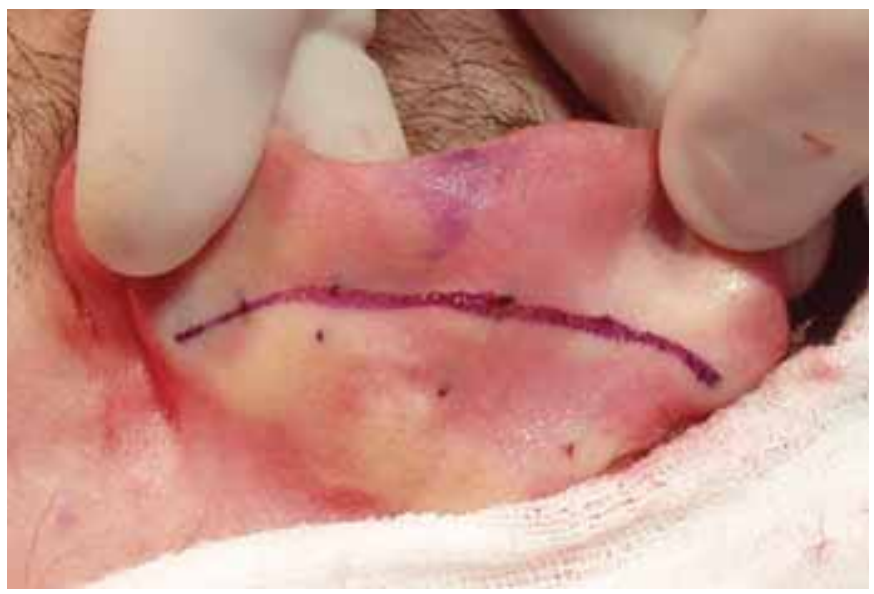




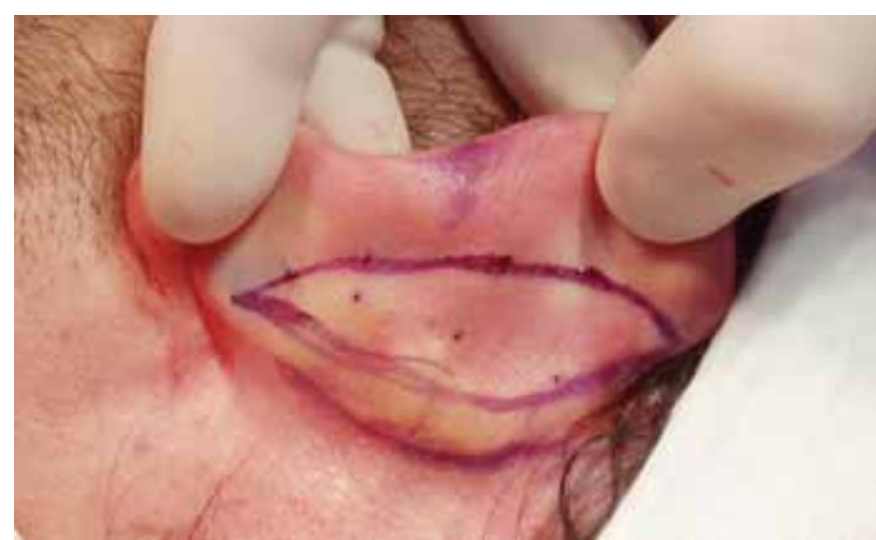

Fig. 8. Marcaje del borde interno posterior a $5 \mathrm{~mm}$ del vértice del ángulo mastoideo-conchal.

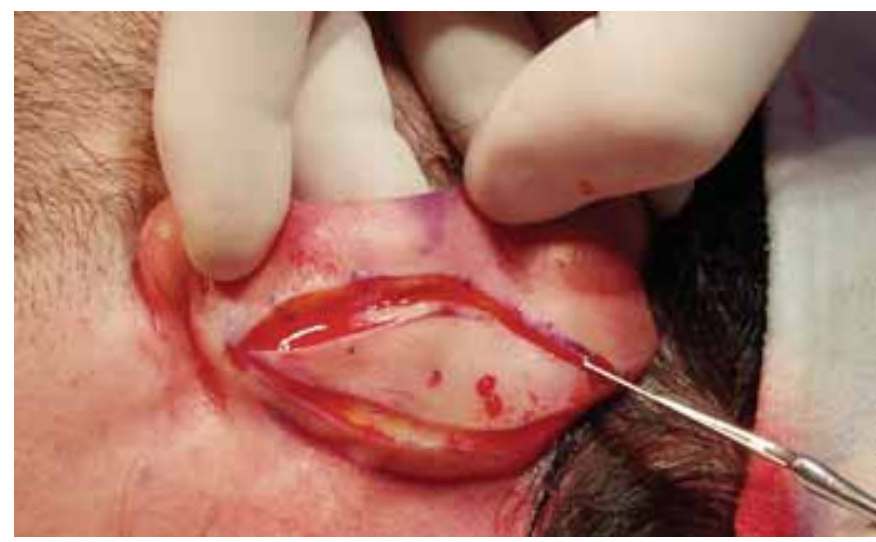

Fig. 10. Incisiones sobre las marcas.

Nuestro abordaje mínimo lo realizamos de la siguiente manera. Sobre una aguja $\mathrm{N}^{\circ} 21 \mathrm{G}$ de 1.5 pulgadas realizamos una angulación de $45^{\circ}$ a $50^{\circ}$ en la punta de su bisel (Fig. 14 y 15) y una vez visualizada el área que hemos de despegar, procedemos al despegamiento mediante disección en el espacio piel-cartílago con el bisel paralelo al cartílago del pabellón auricular y después hacemos cortes-incisión en el cartílago del pabellón auricular anterior, en el área marcada para la formación del neoantihélix, utilizando la aguja con el bisel perpendicular al plano cartilaginoso (Fig. 16).

A continuación flexionamos el cartílago auricular con una ligera presión sobre el tubérculo de Darwin

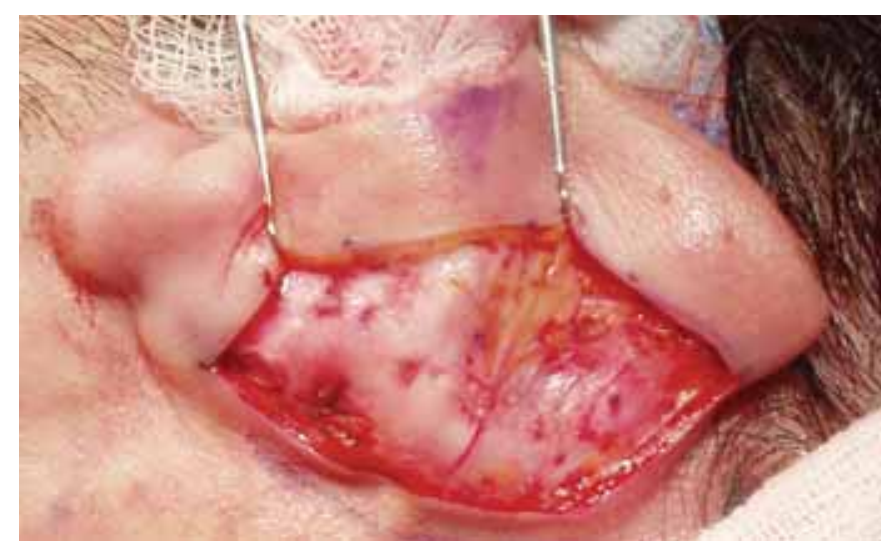

Fig. 12. Pericondrio expuesto.

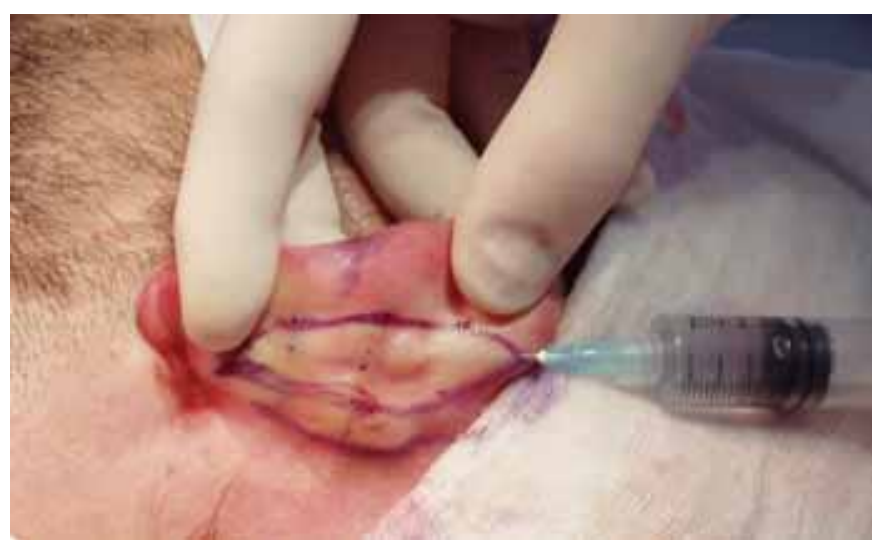

Fig. 9. Hidrodecolamiento con anestésico local.

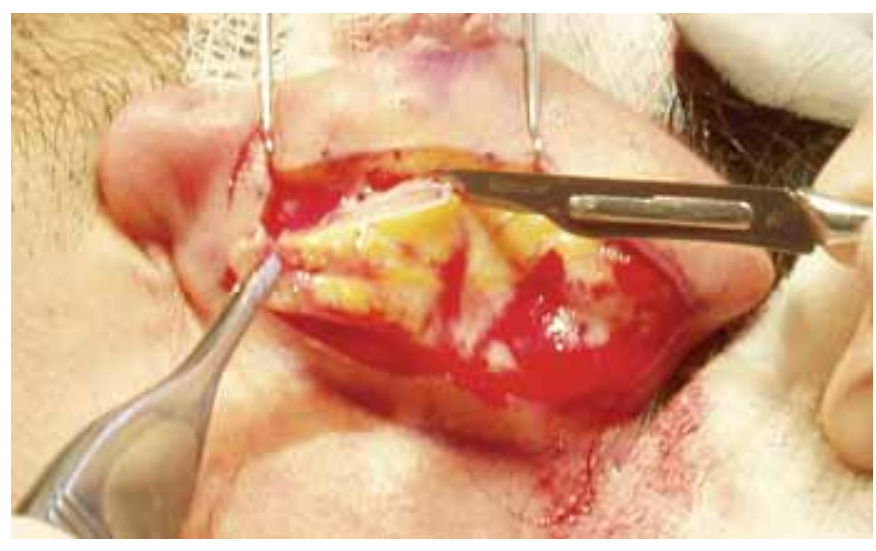

Fig. 11. Resección cutánea y de celular subcutáneo hasta pericondrio.

para comprobar que con los cortes de relajación realizados se obtiene la flexibilidad y la forma natural del neoantihélix que deseamos (Fig. 17).

Finalmente, colocamos de 2 a 3 puntos simples en el pericondrio del pabellón auricular posterior con monofilamento absorbible $4 / 0$, formando la curvatura anatómica del neoantihélix (Fig. 18) y suturamos la piel con monofilamento no absorbible de nylon 4/0.

El resultado es inmediato (Fig. 19, 20): un neoantihélix y simetría entre los dos pabellones auriculares. Curamos con ungüento antibiótico y ferulizamos con tiras de algodón húmedo que se mantienen durante 72 horas.

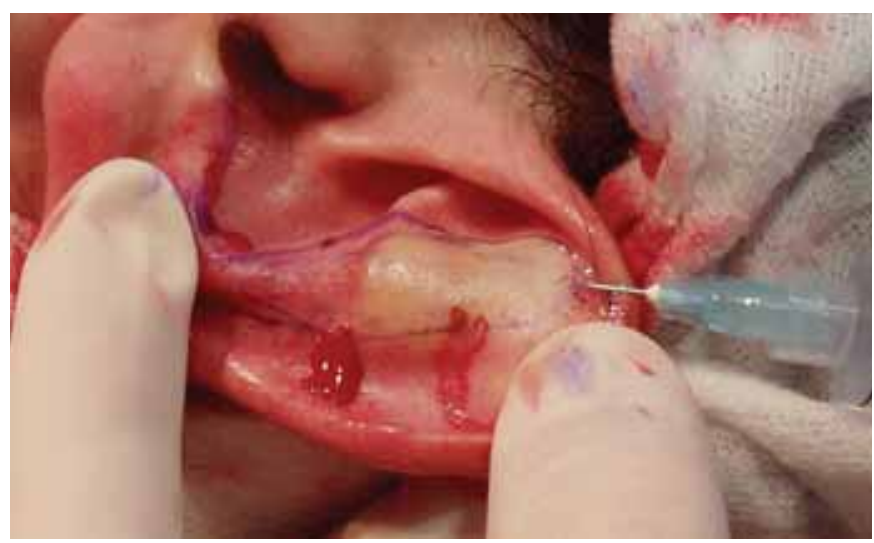

Fig. 13. Infiltración con anestésico local para decolamiento del antihélix anterior. 

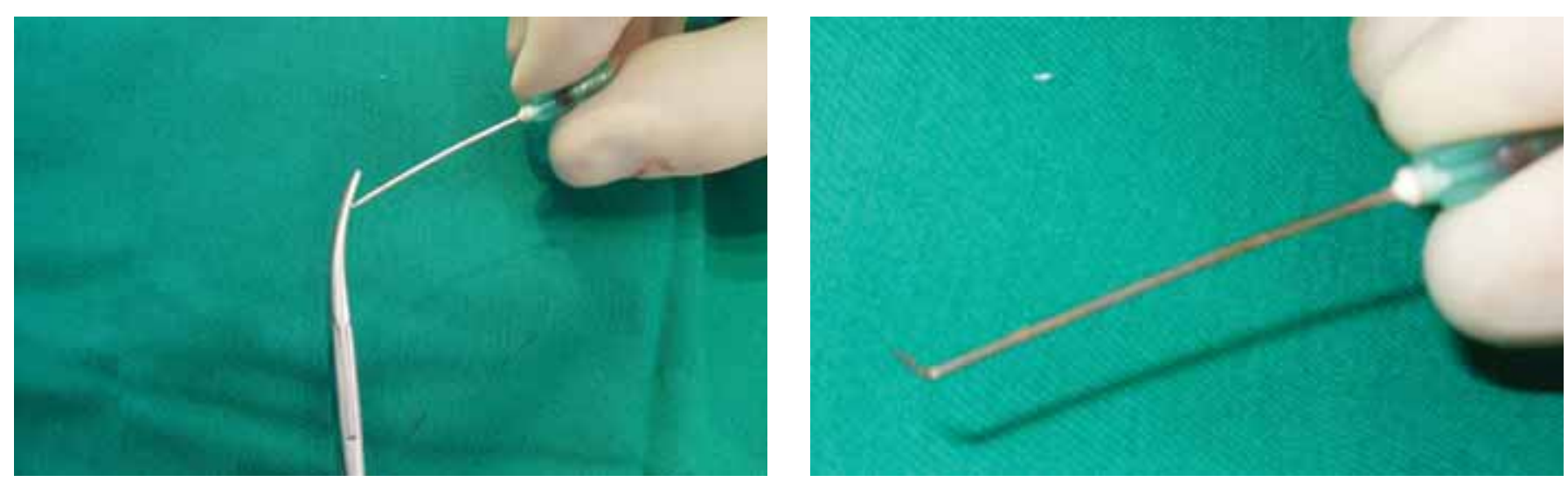

Fig. 14-15. Manipulación de una aguja no 21 G de 1,5 pulgadas para angular 45-50 la punta en bisel.
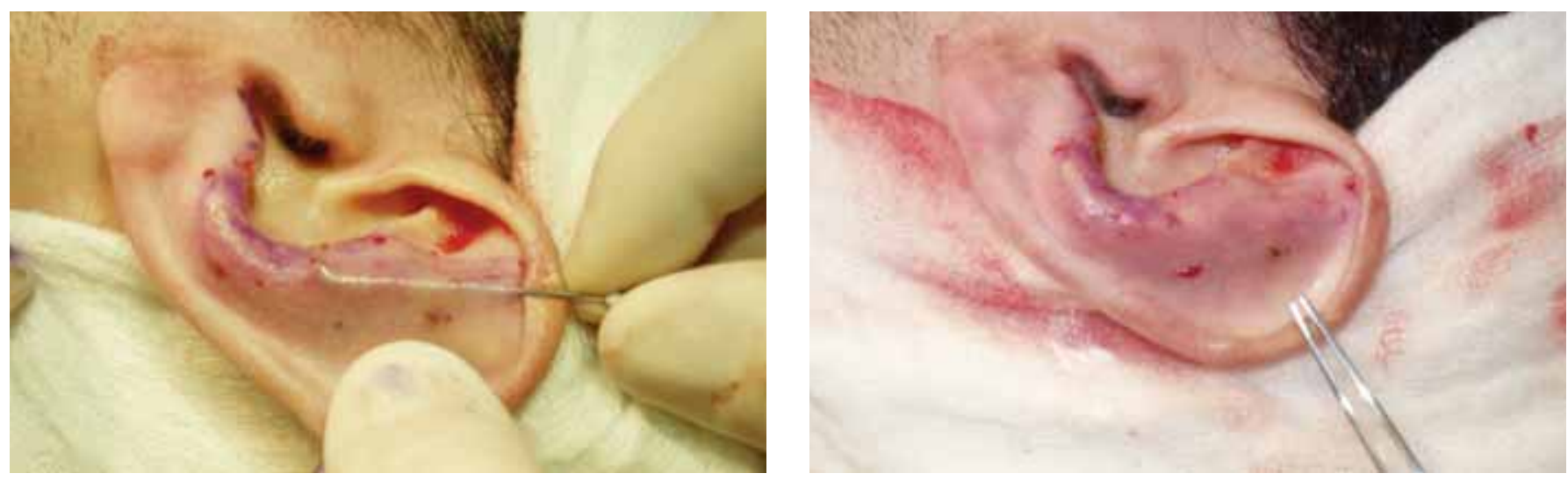

Fig. 16-17. Abordaje mínimo: disección del espacio piel-cartílago con la aguja angulada con el bisel paralelo al cartílago auricular y cortes-incisión en la superficie anterior del cartílago, en dirección vertical y con el bisel perpendicular al plano cartilaginoso.

\section{Discusión}

Las técnicas empleadas para el tratamiento de orejas prominentes son múltiples y variadas. Dieffenbach intenta corregir las orejas prominentes con la resección de un huso cutáneo retroauricular (15), manteniéndose desde entonces su técnica en todos los procedimientos descritos. A excepción de la técnica en isla descrita por Pitanguy, en la que se escinde un huso delgado y largo de cartílago y se proyecta hacia delante para crear el esqueleto del antihélix (5), todos los demás procedimientos operan sobre el cartílago

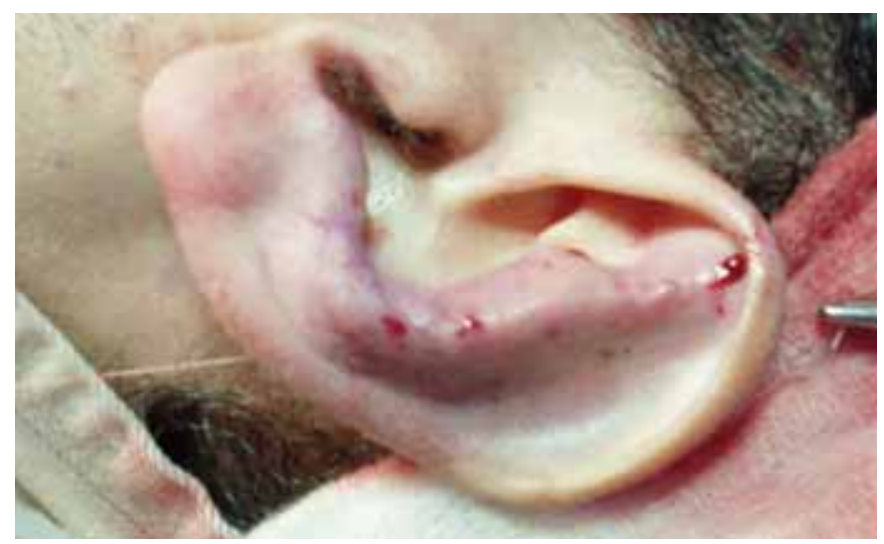

Fig. 18. Presión sobre el tubérculo de Darwin para comprobar flexibilidad del cartílago y forma del neoantihélix. auricular mediante cortes o afinamiento anterior o posterior para formar un antihélix al plegar, debilitar o relajar el cartílago del pabellón auricular, con una visión directa del cartílago manipulado.

Converse incide el cartílago en el área posterior del antihélix y lo fija mediante sutura no absorbible (16); Mustardé, con una incisión por debajo y paralela al hélix, sutura a través del cartílago y el pericondrio, sin tocar la piel ventral para plegar un antihélix (17); Stenström utiliza una escofina para producir un adelgazamiento del cartílago posterior (18), mientras que Weerda lo hace con una broca diamantada (7); Cuenca-Guerra emplea una legra curva que debilita las caras anterior y posterior del cartílago (4); Walter expone y libera previamente la cara anterior del cartílago del pabellón auricular y con múltiples cortes transversales en el área de proyección antihelical, logra debilitar el cartílago (7).

Nuestro procedimiento emplea una aguja con punta angulada para decolar la piel del cartílago del pabellón anterior en el área correspondiente al neoantihélix, creando un bolsillo a diferencia de las otras técnicas que realizan incisiones y decolan ampliamente la piel que cubre el cartílago auricular.

Además, con el mismo instrumento, se realizan fácilmente múltiples incisiones verticales al eje 


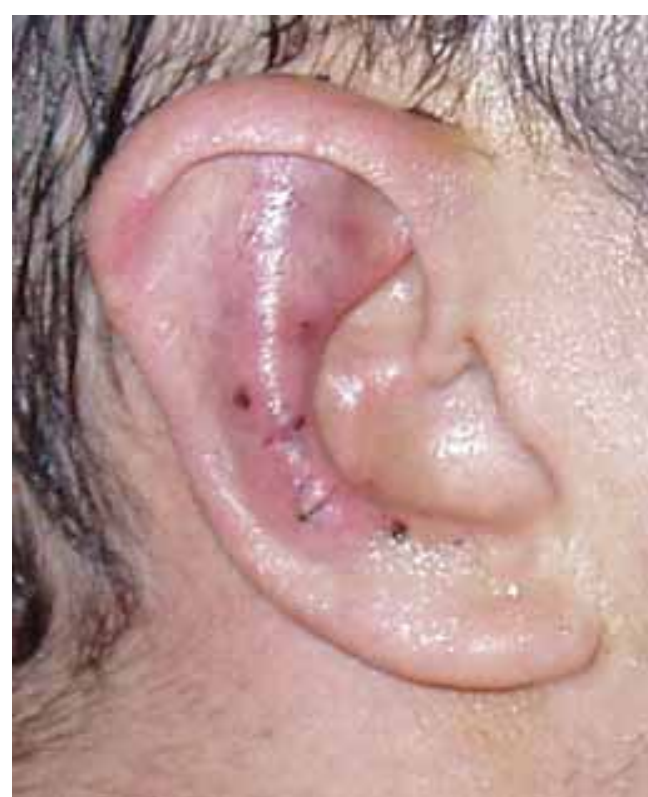

Fig. 19-20. Resultado inmediato.

mayor del área de proyección del neoantihélix, conformándo la curvatura normal y anatómica del pabellón auricular.

Mediante puntos de sutura absorbible colocados en el pabellón auricular posterior doblamos el mismo, produciendo en el cartílago el pliegue o curvatura deseada del neoantihélix, al igual que hacen otras técnicas.

En relación a las complicaciones de las técnicas tradicionales, vemos que en la de Mustardé se han reportado queloides, granulomas y tendencia a la deformi-

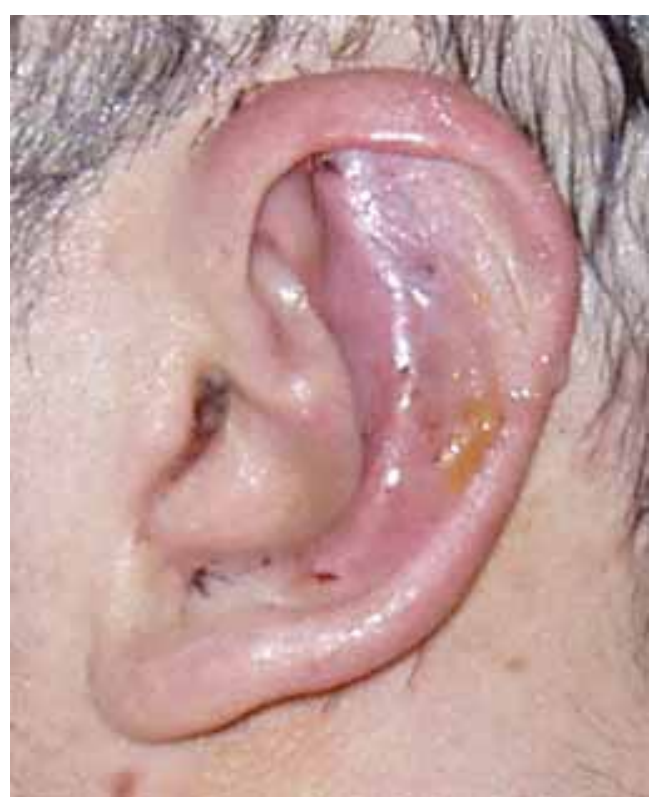

dad original. En las técnicas de Stenström y Weerda, que emplean instrumental abrasivo, se puede observar condronecrosis y fibrosis; Cuenca-Guerra reporta epidermolisis y cicatrices hipertróficas. También se han descrito bordes agudos notorios en el área antihelical con las técnicas de Walter y converse $(7,16)$.

En la técnica descrita en este trabajo, utilizada desde 2004 en más de 50 pacientes de edades comprendidas entre los 4 y los 45 años, hemos observado una mínima presencia de equímosis y edema, así
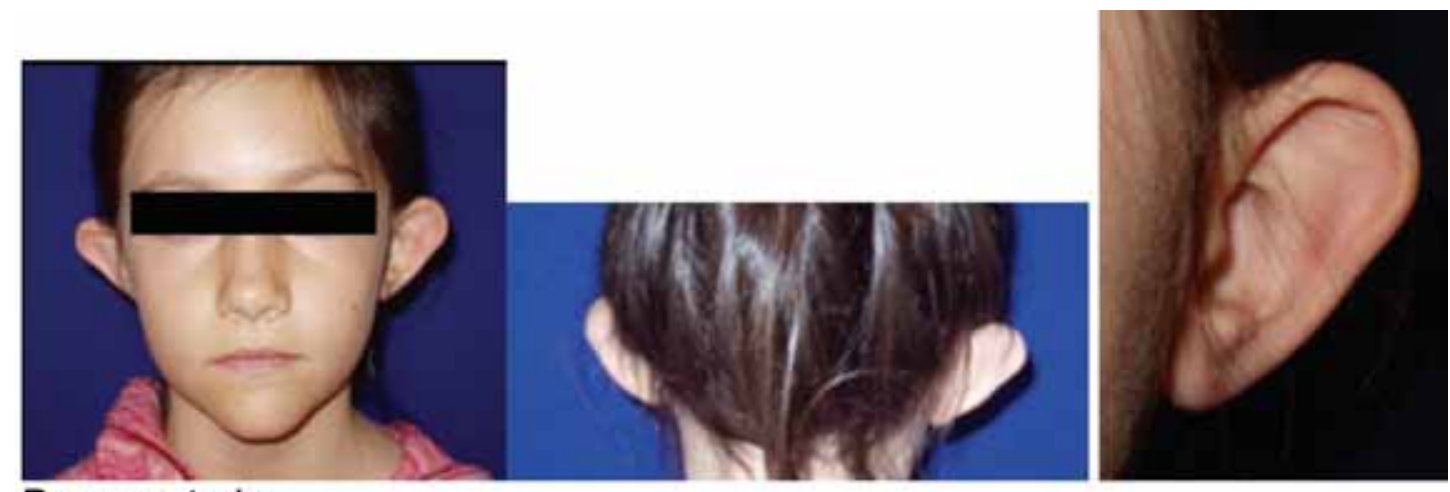

\section{Preoperatorio}
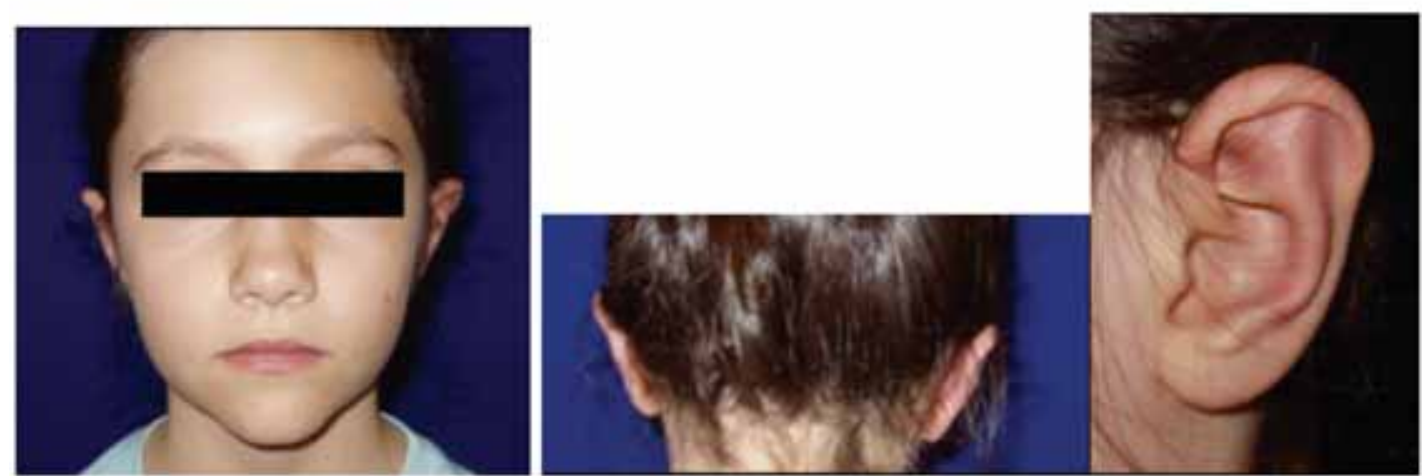

Postoperatorio

Fig. 21. Caso 1. 

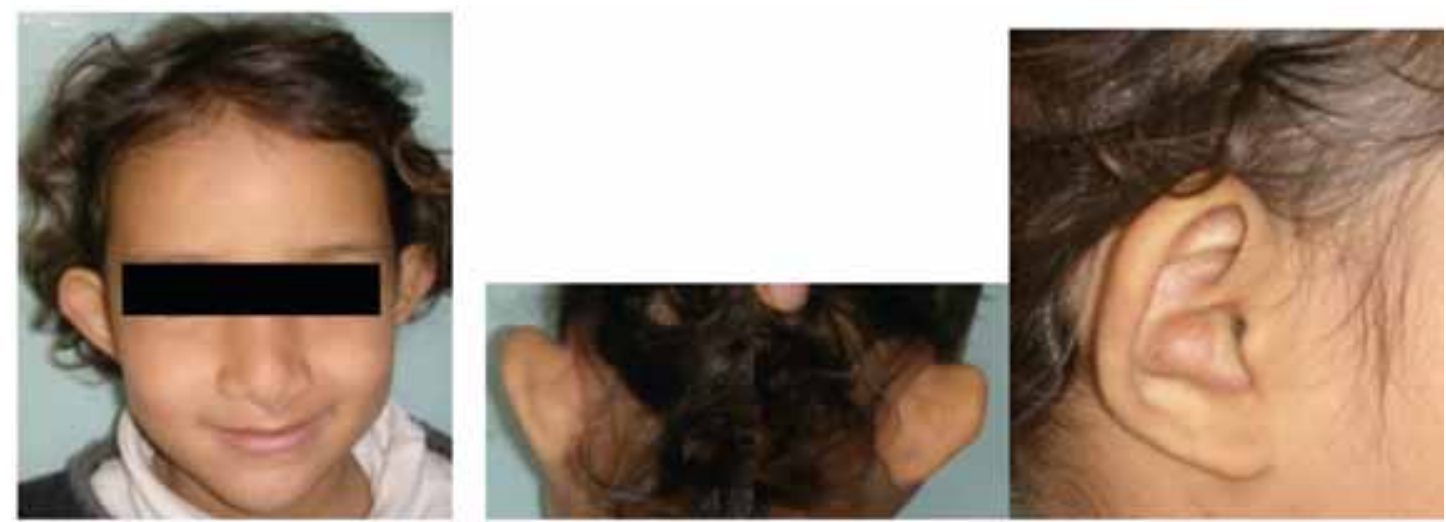

\section{Preoperatorio}

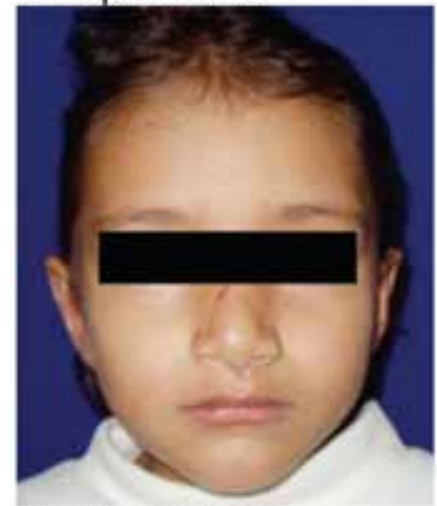

Postoperatorio

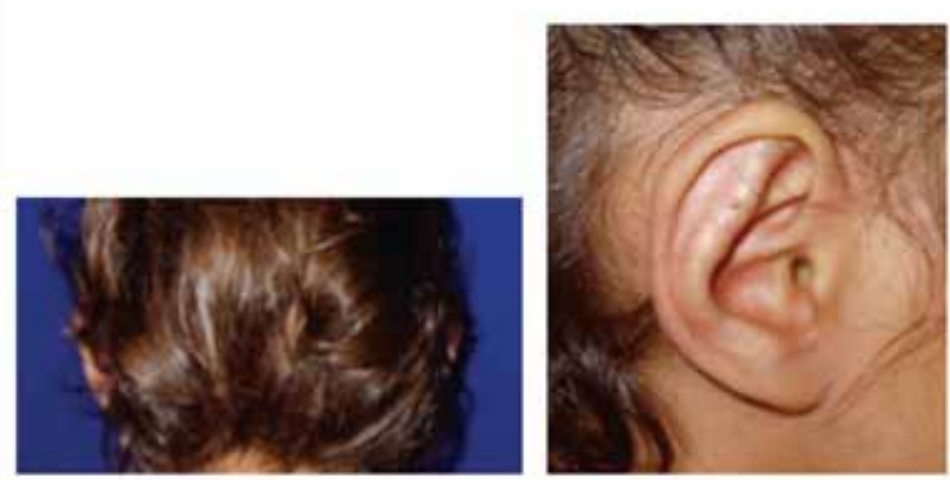

Fig. 22. Caso 2.

como manifestación de dolor leve en pocos casos que cedía con analgésicos anti-inflamatorios no esteroideos. En 3 casos hemos tenido cicatrices hipertróficas en el pabellón auricular posterior: 1 que se solucionó con infiltración intracicatricial con Triamcinolona y 2 que se trataron mediante masajes sobre la cicatriz. En ninguno de nuestros casos hemos observado recidiva ni necrosis de piel (Fig. 21, 22).

A medida que mejora la curva de aprendizaje de este procedimiento quirúrgico, el tiempo promedio de ejecución de la técnica en una oreja es de aproximadamente 20 minutos, por su facilidad. Es recomendable realizar el procedimiento en ambas orejas para conseguir una simetría completa.

\section{Conclusiones}

El procedimiento quirúrgico que presentamos es una modificación técnica de fácil ejecución para la corrección quirúrgica de las orejas prominentes, que se aplica en pacientes desde los 4 años de edad en adelante; con un instrumento simple realizamos cortes verticales de relajación en el pabellón auricular anterior en el área que corresponde al antihélix, con lo que moldeamos y formamos el neoantihélix. La piel anterior del pabellón auricular se acomoda fácilmente a la nueva curvatura del cartílago auricular.

Con este procedimiento el transoperatorio es breve y el postoperatorio inmediato transcurre con mínimo dolor, equímosis y edema al tratarse de un abordaje mínimo.

En pocos casos observamos cicatrices hipertróficas en el pabellón auricular posterior, y si se producen se solucionan en pocos meses con masaje y/o aplicación de cremas con corticoides. No hemos tenido casos de recidiva.

\section{Dirección del autor}

Dr. Pablo Dávalos Dávalos.

Instituto de Cirugía Plástica. Villalengua Oe4-319, Quito, Ecuador.

e-mail: pablo_davalos@hotmail.com

Bibliografía

1. Thorek P.: "Anatomy in Surgery". $2^{\mathrm{a}}$ ed.- USA. J.B. Lippincott Co.1962. Pp. 98-99.

2. Corzo Sosa A, Castañeda Guerra A, Grajeda López P.: "Otoplastia directa: Colgajo pericondrio-cutáneo". Revista de la Asociación Mexicana de Cirugía Plástica, Estética y Reconstructiva, 2000; 10(1):16.

3. Del Vecchyo C, Ugalde A.: "Otoplastia: Técnica personal". Cir. plást iberolatinoam 2004, 30(3): 217.

4. Cuenca-Guerra R.: "Oreja prominente. Plastia mínimamente invasiva”. Revista de la Asociación Mexicana de Cirugía Plástica, Estética y Reconstructiva 1999; 9(3): 120.

5. Pitanguy I, Rebello C.: "Orelhas en abano. Consideraciones em torno do problema e sugestao de técnica pessoal". Rev. Bras. Cir. 1961, 42: 267.

6. Escudero Nafs FJ: "Corrección de las orejas prominentes mediante Otoplastia de Chongchet. Ventajas e Inconvenientes". Revista de divulgación científica de la AECEP, 2007, 2: 42. 
7. Naumann A.: "Otoplasty-techniques, characteristics and risk". GMS Current Topics in Otorhinolaryngology-Head and Neck Surgery 2007, 6: 1 .

8. Guyuron B, De Luca L.: "Ear Projection and the Posterior Auricular Muscle Insertion”. Plast. Reconstr. Surg. 1997, 100(2): 457.

9. Manstein C.: "Ear, Congenital Deformities" [emedicine] [actualizado Mayo 31, 2005]. Disponible en: www.emedicine.com/ ent/topic79.htm

10. Bisaccia E, Lugo A, Johnson B, Scarborough D.: "Otoplasty: The Surgical Approach to protuberant Ears”. Skin \& Aging. 2004, 10(12): 42.

11. Estrada Sarmiento M.: “Orejas prominentes. Evolución postoperatoria”. Rev Cubana Cir 1998, 1(37): 5.

12. Dávalos Dávalos PA.: "Tratamiento de orejas prominentes. Técnica quirúrgica alternativa". Presentación en el XXX Congreso Nacional Sociedad Colombiana de Cirugía Plástica, IV Congreso
Regional Bolivariano de la FILACP, Cartagena de Indicias, Colombia 27-31 Marzo 2005.

13. Sclafani AP. : "Otoplasty". [emedicine] [actualizado Agosto 29, 2006]. Disponible en: www.emedicine.com/ent/TOPIC110.htm

14. Dávalos Dávalos PA: "Orejas prominentes, otoplastia, una alternativa quirúrgica. Presentación en el V Congreso Regional Bolivariano de Cirugía Plástica de la FILACP, Caracas, Venezuela 2-5 Mayo 2007.

15. Dieffenbach JF.: "Die Operative Chirugie". Liepzig, F. A. Brockhaus, 1845

16. Converse J, Nigro A, Wilson F, Johnson N.: “A technique for surgical correction of lop ears" Plast. Reconstr. Surg. 1955, 15: 411.

17. Mustardé JC.: "The correction of prominent ears using simple mattress sutures". Brit J Plast Surg. 1963; 16: 170.

18. Stenström S.: "A "natural" technique for correction of congenitally prominent ears”. Plast Reconstr Surg. 1963; 32: 509.

\title{
Comentario al trabajo ıOtoplastia en orejas prominentes: alternativa quirúrgica»
}

\author{
Dr. Francisco J osé Escudero Nafs. \\ Cirujano Plástico. Servicio de Cirugía Plástica Hospital Universitario Virgen del Camino. Pamplona. España.
}

La causa más frecuente de oreja prominente es el desarrollo insuficiente del pliegue del antihélix. Se han publicado numerosas técnicas para plegar el antihélix. Un grupo se basa en la tendencia del cartílago a doblarse espontáneamente cuando la cara anterior es debilitada mediante rayaduras o raspado, al liberarse la tensión en dicha superficie. Con esta finalidad se han empleado diversos instrumentos, como hojas de bisturí y raspas, entre otros. El Dr. Dávalos y sus colaboradores describen una técnica de otoplastia en la que aportan, para simplificar el procedimiento, el empleo de una aguja de calibre 21 con punta angulada para la realización de cortes de relajación.

El uso de una aguja para rayar la superficie del antihélix se remonta, al menos, a 1979, cuando Vecchione lo publica como idea e innovación en la revista Plastic and Reconstructive Surgery (1). Una aguja intravenosa de calibre 18 era introducida directamente a través de la piel anterior y ubicada en un túnel subpericóndrico a lo largo del nuevo pliegue propuesto. Con la superficie cortante de la punta rayaba el cartílago hasta obtener el pliegue deseado. Posteriormente otros cirujanos han descrito modificaciones en la aguja para facilitar el rayado del cartílago. Mahler (2), Peled (3) y Burres (4) han empleado una aguja con la punta doblada. Mientras estos autores doblaban la punta de la aguja en un eje, Bulstrode et al (5) describieron un método para doblar la punta de una aguja hipodérmica en dos angulaciones, de modo que obtenían una fina punta orientada verticalmente. Esta aguja era introducida y avanzada con la punta paralela a la superficie del cartílago y entonces girada $90^{\circ}$ para colocarla perpendicularmente al plano cartilaginoso, el cual era rayado hasta obtener el pliegue deseado.

El Dr. Dávalos y colaboradores refieren que con el empleo de la aguja angulada logran fácilmente la formación del neoantihélix y de un ángulo mastoideo conchal de $30^{\circ}$. Sin embargo, considero que para la corrección de dicho ángulo se precisan procedimientos complementarios, como las suturas conchomastoideas.

Una ventaja destacable del rayado anterior percutáneo mediante aguja es que requiere una disección mínima del cartílago, a diferencia de la técnica de Chongchet, en la cual el cartílago es ampliamente expuesto para proceder a la realización de rayaduras con hoja de bisturí. Sin embargo, este abordaje, que es el que empleo habitualmente, permite desarrollar el pliegue del antihélix bajo visión directa, lo cual es importante para controlar con precisión la curvatura y profundidad de los cortes.

Las técnicas quirúrgicas descritas y empleadas para la corrección de las orejas prominentes son numerosas y, como bien dicen el Dr. Dávalos y sus colaboradores, el cirujano debe utilizar aquella con la que mejores resultados obtenga.

Bibliografía

1. Vecchione TR.: "Needle scoring of the anterior surface of the cartilage in otoplasty". Plast Reconstr Surg. 1979; 64: 568.

2. Mahler D.: "The correction of the prominent ear". Aesth Plast Surg. 1986; 10: 29.

3. Peled IJ.: "Knifeless otoplasty: how simple can it be?". Aesth Plast Surg. 1995; 19: 253.

4. Burres S.: "The anterior-posterior otoplasty". Arch Otolaryngol Head Neck Surg. 1998; 124: 181.

5. Bulstrode NW, Huang S, Martin DL.: "Otoplasty by percutaneous anterior scoring. Another twist to the story: a long-term study of 114 patients”. Br J Plast Surg. 2003; 56: 145.

\section{Respuesta al comentario del Dr. Francisco José Escudero Nafs}

\section{Dr. Pablo A. Dávalos Dávalos}

Agradezco el comentario del Dr. Escudero, quien ha profundizado en el origen de la otoplastia con rayadura de cartílago en pabellón auricular anterior, empleando una aguja como instrumento. Menciona otros autores y compara la técnica descrita con la otoplastia abierta de abordaje posterior y liberación anterior del cartílago auricular, técnica de Chongchet, donde se expone el cartílago, se visualiza y se realizan los cortes controlando su curvatura y profundidad. En nuestro artículo, tal como Vecchione, podemos afirmar que la profundidad, anchura y corte pueden ser ajustados para obtener la laxitud correcta en el nuevo pliegue.

El Dr. Escudero también considera que para corregir el ángulo mastoideo conchal se requieren además suturas conchomastoideas. Sobre este aspecto diremos que en la técnica por nosotros realizada no son necesarias, porque es suficiente con colocar dos o tres puntos en el pericondrio posterior con sutura monofilamento absorbible, con lo que logramos plegar, asegurar y formar el neoantihélix y con la sutura de los bordes cutáneos del huso auricular posterior, se refuerza además la curvatura del neoantihélix y se forma el ángulo deseado al apoyarse en el borde cutáneo próximo al vértice.

Los objetivos mencionados por Mahler son tomados en cuenta en nuestra publicación. Es decir, la corrección de la ausencia del antihélix y la corrección del alejamiento de la concha desde la mastoides.

Nuestro enunciado es simplificar un procedimiento quirúrgico, siendo de fácil ejecución, aplicable en niños y adultos, con buenos resultados estéticos y con una baja morbilidad, sin desmerecer las bondades de otras técnicas quirúrgicas descritas. 